\title{
Gestión integrada de recursos hídricos en Uruguay en el contexto internacional
}

\author{
Integrated water resources management in Uruguay \\ within the international context
}

\author{
Míguez, Diana (1) \\ (1) Programa Aguas, Proyectos Ambientales, Gerencia I+D+I, Laboratorio Tecnológico del Uruguay, LATU. \\ Contacto: dmiguez@latu.org.uy
}

RECIBIDO: 9/3/2015 - APROBADO: 17/11/2015

\begin{abstract}
Resumen
La necesidad de proteger el medio ambiente y en especial la cantidad y la calidad de las fuentes de agua dulce contra los efectos de las presiones naturales y antrópicas se ha vuelto cada vez más importante a nivel mundial. Para ello, no basta con aplicar tecnologías de extracción de agua y de depuración efectivas, sino que también se deberían implementar sistemas de gestión participativos para alinear las actividades humanas hacia el desarrollo sostenible. La herramienta más idónea para realizarlo es la Gestión Integrada de Recursos Hídricos (GIRH), mediante la cual se genera información sobre la que basar políticas de gestión ambiental y de provisión de agua potable y saneamiento equitativas, que contemplen las opiniones de todos los grupos de interés. En el caso de Uruguay, la Constitución de la República consagra el derecho de todos los habitantes del país al acceso al agua y al saneamiento. El proceso de implementación del sistema de GIRH se inició dándole participación a la ciudadanía a través de un referéndum, luego del cual los referentes en el tema de todos los sectores de la sociedad aconsejaron a nivel parlamentario, y el procesó derivó en la reglamentación de la ley del agua. Se consolidó así una estructura de gestión por cuencas hidrográficas basada en el ciclo hidrológico que se encuentra hoy en un grado de desarrollo que refleja la mejora continua de la institucionalidad de la gobernanza del agua. En esta revisión se releva el desarrollo de estos procesos tanto a nivel nacional como mundial y se propone una serie de herramientas y estrategias para hacer cada vez más eficiente la gobernanza.

Palabras clave: Gestión Integrada de Recursos Hídricos, gobernanza, comités de cuenca.
\end{abstract}

\begin{abstract}
The need to protect the environment and especially the quantity and quality of the freshwater sources against natural and anthropic pressures has become more and more relevant at a global level. To do so, it is not enough to apply effective water abstraction and depuration technologies, but also to implement participatory management systems to align human activities towards sustainable development. The best fit tool to do so is the Integrated Water Resources Management (IWRM), because it generates information on which equitable environmental management and water provision policies can be set, taking into consideration the opinions of all stakeholders. In Uruguay, the Constitution of the Republic mandates to respect the right of all of the inhabitants in the country to water and sanitation access. The process of implementation of the IWRM in Uruguay initiated by allowing citizens to participate through a referendum, and then the experts in the field belonging to every sector in society advised at a parliament level, ending in the regulation of the Water Law. A management structure based on hydrographic basins and the hydrological cycle was consolidated. Its current degree of development reflects the continuous improvement of the institutional character of water governance. In this work, the development of these processes both at a national and at a global level is reviewed and a set of strategies and tolls to make it more efficient are proposed. Keywords: Integrated Water Resource Management, governance, basin committees.
\end{abstract}




\section{Tabla de contenidos}

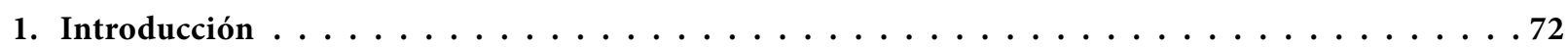

2. Identificación de las amenazas sobre la subsistencia del recurso agua . . . . . . . . . . . . . 73 2.1. Percepción de los países con respecto a la crisis hídrica en base a la ponderación de riesgos globales . . . 73 2.2. Actividades productivas e impactos sobre el recurso agua . . . . . . . . . . . . . . . 73

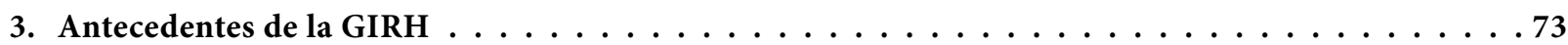

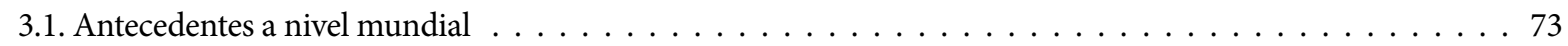
3.2. Situación actual y perspectivas futuras $\ldots \ldots \ldots \ldots \ldots \ldots \ldots \ldots \ldots$ 3.3. Implementación de la gestión por cuencas hidrográficas en el contexto internacional $\ldots \ldots \ldots$. . . . . 74

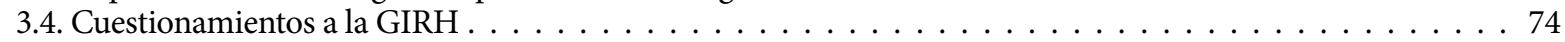

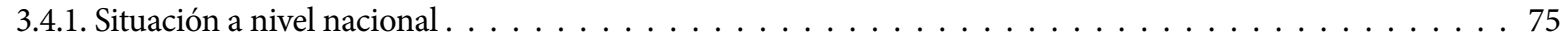

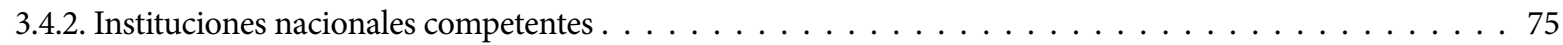
3.4.3. Avances nacionales en la GIRH . . . . . . . . . . . . . . . . . . . . . . . . . . . . 75

4. Herramientas y tecnologías requeridas para la sostenibilidad de los procesos productivos . . . . 76

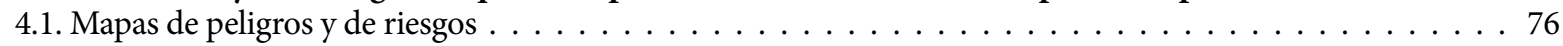

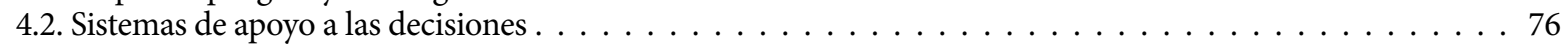

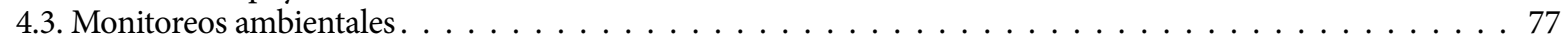

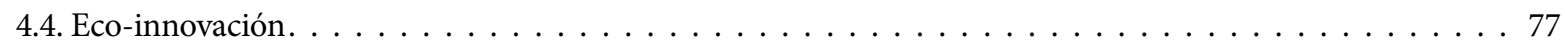

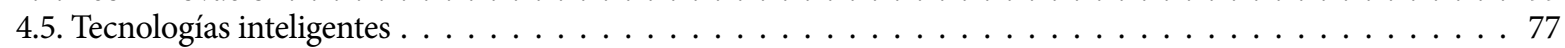

4.6. Nuevas tendencias en indicadores . . . . . . . . . . . . . . . . . . . . . . 77

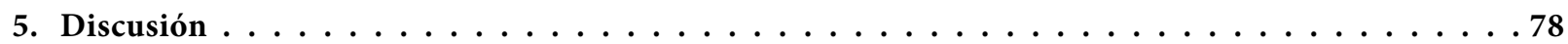

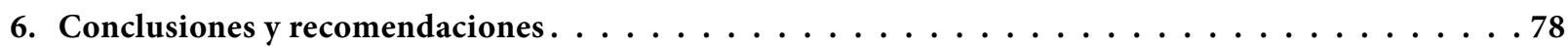

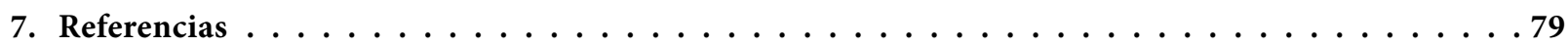

\section{Introducción}

El agua, en tanto recurso natural y bien económico, es parte integral de los ecosistemas y fundamental para la vida humana. Su valor ha sido atesorado desde la Antigüedad; se remonta a la aplicación de las tecnologías de riego y acueductos utilizados como defensa ante eventos de inundación o sequía iniciadas probablemente por los pueblos asirios alrededor del año 691 A.D., seguidos por los egipcios, los griegos, los romanos (Lofrano, et al., 2012) y los pueblos precolombinos (Díaz y Freire, 2008). Según las Naciones Unidas (Naciones Unidas, 2014a; 2014b), entre las mayores presiones que experimenta la disponibilidad de agua a nivel mundial se puede mencionar el aumento de población, que se predice que alcanzará a 9.300 millones en 2050. Según esta fuente, actualmente 2.000 millones carecen de acceso a agua potable y 2.500 millones de personas no tienen acceso a saneamiento. Los intereses contrapuestos de diversos actores de la sociedad por el uso del agua se pueden ejemplificar en la necesidad creciente de producción de alimentos. En el mundo 870 millones de personas sufren desnutrición por dificultad al acceso económico de los alimentos $y$, en algunos lugares, por la escasez de estos alimentos. No toda la producción agrícola es destinada a la alimentación; gran parte es destinada para producir fibras, biocombustibles y alimento para animales. En total, la agricultura de regadío representa el $70 \%$ de las extracciones de agua dulce a escala mundial, a lo que se suman los usos para la distribución de agua potable y para otros fines.

Hacia el año 2025 se estima que 1.800 millones de personas vivirán en regiones con escasez de agua y dos tercios de la población mundial residirá bajo condiciones de estrés hídrico. Esto dirigirá los conflictos hacia la competencia por el agua y propiciará enfermedades debidas a alteraciones en la calidad del agua, así como también se experimentarán pérdidas de biodiversidad y de los servicios de los ecosistemas. Por esto, la comunidad internacional está urgiendo a reformar las políticas y leyes nacionales. Por ejemplo, las Naciones Unidas ha aconsejado a sus Estados Miembro a priorizar el agua, dada su importancia tanto para el ser humano como para el medio ambiente, en la agenda post 2015 de Objetivos del Desarrollo Sostenible (Naciones Unidas, 2015).

A nivel mundial, se considera que, a pesar de la importancia real que representan la escasez y la contaminación del agua, la actual crisis del agua responde primordialmente a una crisis de gobernanza. La gobernanza es definida por la Real Academia Española (2001) como el «arte o manera de gobernar que se propone como objetivo el logro de un desarrollo económico, social e institucional duradero, promoviendo un sano equilibrio entre el Estado, la sociedad civil y el mercado de la economía».

La gobernanza está conformada por los sistemas políticos, sociales, económicos y administrativos requeridos 
para desarrollar y gestionar los recursos hídricos y entregar servicios de agua a los diferentes niveles de la sociedad. Debe reconciliar enfoques y filosofías a veces dispares, lo cual lleva tiempo y depende de la decisión política, las oportunidades y el liderazgo de las instituciones competentes. Es clave para proveer una gestión eficiente de los recursos hídricos que contenga los siguientes componentes: capacidad, políticas, leyes e instituciones. Esto se puede lograr a través de la Gestión Integrada de Recursos Hídricos (GIRH), la cual fue definida por el Comité Técnico de la Asociación Mundial para el Agua (Global Water Partnership Technical Advisory Committee, 2000) como «un proceso que promueve la gestión y desarrollo coordinado del agua, la tierra y los recursos relacionados, con el fin de maximizar el bienestar social y económico resultante de manera equitativa, sin comprometer la sostenibilidad de los ecosistemas».

El desarrollo sostenible apunta a realizar los procesos y actividades humanas minimizando los impactos sobre la disponibilidad y calidad del recurso agua para no comprometer la subsistencia de los habitantes actuales del planeta y de las futuras generaciones.

Las aplicaciones de la GIRH se constatan en la mediación en conflictos transfronterizos, en el otorgamiento de derechos de uso, y en el hecho de incentivar la toma de conciencia sobre las responsabilidades compartidas en cuanto a contaminación y disminución de riesgos sanitarios. Asimismo, provee información de primera mano a los tomadores de decisiones sobre las opiniones y necesidades de todos los grupos de interés, lo cual es útil en la generación de políticas de gestión ambiental $\mathrm{y}$ de agua potable y saneamiento.

\section{Identificación de las amenazas sobre la subsistencia del recurso agua}

\subsection{Percepción de los países con respecto a la crisis hídrica en base a la ponderación de riesgos globales}

La Organización Mundial de la Salud define riesgo como «la probabilidad de ocurrencia de un efecto adverso en un organismo, sistema o sub-población causado bajo circunstancias específicas tras exposición a un agente» (World Health Organization, 2004). Sin embargo, algunos autores en el campo de las Ciencias Sociales, tales como Aven y Renn (2009), se refieren al riesgo como «la incertidumbre sobre y la severidad de las consecuencias (o resultados) de una actividad con respecto a algo que los seres humanos valoran». En esta última interpretación se enmarca la utilizada por el Foro Económico Mundial, que define riesgo global como «un evento o condición inciertos que, de ocurrir, pueden tener un importante efecto negativo para varios países o sectores en los siguientes diez años». Esta ponderación de los riesgos puede orientar hacia la generación de políticas, brindando una visión general de los puntos más críticos de acuerdo a la percepción mundial. En la encuesta mundial del año 2015, se categorizó a las crisis del agua como el factor número 1 en términos de impacto potencial (Water Economic Forum, 2015).

\subsection{Actividades productivas \\ e impactos sobre el recurso agua}

Luego del advenimiento de la industrialización y la agricultura a mayor escala, la mecanización, el comercio y la colonización, la competencia por los recursos se incrementó y se afectaron la calidad y la cantidad del agua. Durante la Revolución Industrial, el agua se utilizaba no solamente por las máquinas de vapor, sino también en la agricultura y en el transporte. El impacto económico de su uso en los procesos productivos fue dramático; luego de que se abriera el primer canal en 1761 en Bretaña, bajó el precio del carbón a la mitad causando que en 1815 hubiera más de 2.000 millas de canales para llevar toneladas de materias primas y productos manufacturados (White, 2015). Sin embargo, paradojalmente, los riesgos por enfermedades transmitidas por el agua se incrementaron, dado que el crecimiento económico acelerado no correlacionó con la mejora de la calidad y expectativa de vida de la población (Szreter, 1997).

Después de la agricultura, los mayores usuarios del agua son la industria y la energía (Programa Mundial de Evaluación de los Recursos Hídricos, 2014, Naciones Unidas, 2014b). A esto se agrega el factor del cambio climático, el cual impacta en los indicadores de gestión de la agricultura e impulsa el reconocimiento de los principios éticos comunes a nivel mundial y local (Rossi, 2015). La agricultura, a su vez, es una de las principales responsables de contaminación difusa del agua por nitratos, fosfatos y agroquímicos, en tanto la industria y los usos urbanos son las principales fuentes puntuales de contaminación si los efluentes son vertidos hacia cursos de agua sin tratamientos adecuados (Santos Pereira, et al., 2009).

\section{Antecedentes de la GIRH}

Con el correr de los años el cúmulo de situaciones consignadas en cuanto a las amenazas para el agua y la subsistencia del planeta llevó a la humanidad a reflexionar sobre las estructuras y forma de gestionar el agua, cambiando el enfoque y entendiendo el agua como parte integral de un ecosistema dinámico. Se percibió que las presiones crecientes sobre los recursos hídricos en cuanto a demanda y vulnerabilidad de los recursos requerían una gestión holística del agua, lo cual favoreció el posicionamiento de la Gestión Integrada de los Recursos Hídricos (GIRH) como alternativa aceptada internacionalmente hacia un desarrollo y gestión eficientes, equitativos y sostenibles. Este concepto ha progresado históricamente.

\subsection{Antecedentes a nivel mundial}

En el año 1933 se estableció la Autoridad del Valle del Tennessee para la gestión de los diversos usos del recurso hídrico, pero estas actividades aún no se realizaban en forma coordinada. Posteriormente, el discurso internacional en la temática enunció una serie de declaraciones y documentos, tales como la Visión Mundial para el Agua, la primera conferencia global en Mar del Plata, de 1977, en la que se expresa esta necesidad de coordinación.

Sin embargo, fue después de la Agenda 21 y de la Cumbre Mundial sobre Desarrollo Sostenible en 1992 en Río de Janeiro cuando el concepto de GIRH fue objeto de profundos debates 
que incluían sus implicaciones en la práctica, en contextos como la Comisión Mundial de Represas, los Objetivos de Desarrollo del Milenio y los resultados de la Comisión del Desarrollo Sostenible de las Naciones Unidas, las declaraciones de Dublín y de París sobre Agua y Desarrollo y la declaración ministerial del Foro Mundial del Agua.

En la declaración de Dublín sobre el agua y el desarrollo sostenible de 1992 -enunciada en la reunión técnica previa a la Conferencia de las Naciones Unidas sobre el Medio Ambiente y el Desarrollo (CNUMAD) celebrada en Río de Janeiro en junio de 1992- se refiere a la vulnerabilidad del agua por su carácter finito y se la valora como esencial para la vida, el desarrollo y el ambiente. Asimismo, se entiende que se requiere un enfoque holístico y participativo que vincule el agua usada en una cuenca y los acuíferos subterráneos. Se propugna entonces la consulta pública y el rol de las mujeres en la gestión, en la salvaguarda y en la provisión del agua, incluyéndola en las decisiones y en la generación de políticas. El agua es también un bien económico, fundamental para la industria y la agricultura, pero siempre que se respete el derecho de cada ser humano a tener agua potable y saneamiento a un precio razonable, a la vez que se proteja la conservación de los recursos y la naturaleza.

\subsection{Situación actual y perspectivas futuras}

Actualmente, las Naciones Unidas han declarado como Decenio Internacional para la Acción «El agua fuente de vida» entre 2005-2015 (Naciones Unidas, 2003), en cuyo marco se contempla el agua como elemento determinante en el desarrollo económico y social y como un recurso natural vital que debe ser tratado.

La declaración de Río+20 compromete a los países a lograr el acceso progresivo al agua y al saneamiento a precios razonables, mejorar la implementación de gestión de recursos hídricos, proteger y gestionar en forma sostenible los ecosistemas, prevenir los desastres ocasionados por cambios en el régimen hídrico y eventos extremos (sequías e inundaciones), reducir significativamente la contaminación del agua y aumentar su calidad, tratar y reutilizar aguas residuales, aumentar la eficiencia en el agua y reducir pérdidas de agua.

La Agenda para el Desarrollo Post 2015 recoge los compromisos anteriores bajo el lema «asegurar agua sostenible para todos", para promover el bienestar humano, la prosperidad económica y la conservación de los recursos ambientales, con los siguientes pilares: agua potable, saneamiento e higiene, recursos acuáticos, gobernanza, desastres relacionados al agua, y contaminación por aguas residuales y calidad de agua. En el marco de la Agenda se abordan las tres dimensiones del desarrollo sostenible, social, económica y ambiental, y se apunta también al cometido de erradicar la extrema pobreza para el año 2030 (Naciones Unidas, 2014a).

La GIRH está basada en los recursos hídricos que integran los ecosistemas y constituyen un recurso natural y un bien social y económico. La competencia por estos recursos requiere la reforma de los sistemas de gobernanza para obtener beneficios sostenibles e igualitarios (Global Water Partnership, 2012). Por tanto, para lograrlo, las herramientas de la Global Water Partnership (GWP) para la GIRH se basan en el establecimiento inicial del entorno, contemplando todas las partes interesadas (individuos e instituciones públicas o privadas, ONGs, hombres y mujeres, sin distinguir su poder económico). En la definición de las políticas se recomienda: tener en cuenta metas para el uso del agua, la protección y la conservación, estimar los valores relativos del ambiente, la economía y los aspectos sociales del agua, y asignarles responsabilidades a los actores públicos y privados, incluyendo a organizaciones de cuenca y reconociendo a la mujer en su rol de usuaria y partícipe fundamental en la gestión del agua.

\subsection{Implementación de la gestión por cuencas hidrográficas en el contexto internacional}

El trabajo en cuencas ha sido incorporado en legislaciones en muchos países (Sudáfrica, Brasil, Unión Europea, Nigeria, Indonesia, Holanda, etcétera). Entre los beneficios potenciales de la GIRH en los países africanos se encuentra la facilitación de un acceso más equitativo al agua en entornos sociales muy complejos, con sobrepoblación y en ocasiones con situaciones de violencia (Programa de las Naciones Unidas para el Medio Ambiente, 2014).

La situación en la región muestra avances en la gestión del agua y del territorio. Sin embargo, en algunos países, como por ejemplo Argentina, falta una visión sistémica y proactividad en los diseños de las políticas (Duek y Comellas, 2011). En Brasil, la gestión de cuencas hidrográficas se ha desarrollado desde la década de 1970. En 1978 se estableció un comité especial de estudios integrados de cuencas hidrográficas (CeeIB, por su sigla en portugués); el proceso desembocó en la organización mandatada por la Ley № 9.433, de 8/1/1997, que dio a Brasil una nueva política de recursos hídricos y organizó el sistema de gestión por cuencas hidrográficas (Porto y La Laina Porto, 2008).

Las lecciones aprendidas de la implementación de la GIRH en Europa indican que una agencia centralizada que represente un único aspecto del uso del agua no debe dominar sino coordinar en forma horizontal a los niveles nacionales, de cuenca y de sub-cuenca, informando e involucrando activamente a los grupos de interés en un ámbito de transparencia y apertura, incluyendo también a los grupos menos privilegiados de la sociedad, sin olvidar la preservación de los ecosistemas y las estrategias de adaptación al cambio climático. La gestión de la demanda del agua debe proveer incentivos económicos tendientes a la conservación del agua y su ahorro. En Europa los siguientes principios generan instrumentos económicos: el que contamina paga; el beneficiario paga; la equidad, y la coherencia entre diversas políticas que impactan el sector hídrico (Organisation for Economic Co-Operation and Development (OECD), Comisión Económica de las Naciones Unidas para Europa. Integrated Water Resources Management in Eastern Europe, the Caucasus and Central Asia, European Union Water Initiative National Policy, 2014).

\subsection{Cuestionamientos a la GIRH}

Si bien los fines de la GIRH son loables, algunos autores han remarcado problemas en su implementación debido a barreras institucionales, de comprensión del concepto y por la complejidad inherente de la cuenca (Grigg, 2008). Según Anzaldi et al. (2014), algunas de las causas serían la falta de herramientas de integración para apoyar las decisiones de gestión y planeamiento y la segmentación de las instituciones responsables del planeamiento y la gestión de los recursos, 
en conjunto con una baja cuota de participación de todos los actores en los procesos de decisión.

En la gestión moderna de los recursos hídricos deben tenerse en cuenta los servicios y los intereses contrapuestos de los individuos, las industrias y la naturaleza, manteniendo la armonía entre quienes hacen uso de recursos compartidos y sistemas que albergarán a las futuras generaciones. La protección de estos servicios debe conectarse al uso de la tierra, por lo cual es preciso anticiparse a los posibles impactos, mientras que la GIRH puede contribuir a la resolución de conflictos.

A pesar de que se reconoce a las instituciones de cuencas por su rol clave en la GIRH, se argumenta que puede ser difícil lograr que exista el suficiente involucramiento público requerido para generar confianza y empoderamiento entre los distintos actores y crear respeto y apoyo para implementar las decisiones. Por tanto, debería existir un mandato claro, con una estrategia a largo plazo y una estructura organizacional establecida, coordinada, no solamente descentralizada, que siga una visión y un plan comunes. También es aconsejable generar alianzas entre beneficiarios, organismos de control y tomadores de decisiones para catalizar los cambios institucionales de implementación de planes de GIRH. No obstante, el control de la gestión del agua se debe conservar en la órbita pública (Iza y Stein, 2009).

\subsubsection{Situación a nivel nacional}

Si bien en el año 2012 Uruguay figuraba entre los países que no estaban realizando una eficiente gestión de recursos hídricos (Naciones Unidas, 2012), recientemente han habido avances en este tema, ya que las instituciones nacionales competentes se encuentran trabajando en GIRH a través de PLANAGUA, un proyecto dirigido por la DINAGUA y financiado por el Banco Interamericano de Desarrollo (BID) y el Fondo Español de Cooperación para Agua y Saneamiento en América Latina y el Caribe (Presidencia de la República Oriental del Uruguay, 2013).

\subsubsection{Instituciones nacionales competentes}

Dentro del Ministerio de Vivienda, Ordenamiento Territorial y Medio Ambiente (MVOTMA), la DINAGUA elabora y propone las políticas públicas del sector, la DINAMA controla la calidad del recurso agua en la naturaleza y, por medio del instrumento de evaluación de impacto ambiental, Decreto $349 / 005$ (Uruguay, 2005), controla proyectos de las plantas de tratamiento de efluentes, y la DINOT establece el ordenamiento territorial. La Dirección Nacional de Hidrografía (DNH), del Ministerio de Transporte y Obras Públicas, se encarga de la gestión de puertos, el dragado de las vías navegables y la regulación de las extracciones de áridos subacuáticos $(\mathrm{DNH}$, 2015). Actualmente, las competencias en materia de evaluación, administración y control de los recursos hídricos fueron transferidas desde la DNH a DINAGUA-MVOTMA por intermedio de la Ley 18.172 del 31 de agosto de 2007 (DINAGUA, 2012).

La URSEA fiscaliza y regula a los prestadores de servicios, en tanto la OSE es responsable del abastecimiento de agua potable en todo el país y de alcantarillado, esto último con excepción de Montevideo.

\subsubsection{Avances nacionales en la GIRH}

Cabe destacar que las cuencas son las áreas físicas donde los procesos naturales se conectan con los socio-económicos y en los cuales el agua interactúa con otros recursos naturales a través del ciclo hidrológico.

Las cuencas de los ríos, en términos geográficos, no necesariamente coinciden con las subterráneas, por lo cual se debe tener en cuenta estos aspectos y el agua en el ciclo del agua y sus relaciones con el aire, los suelos y los sedimentos.

Las provincias hidrogeológicas de nuestro país, determinadas por técnicas hidrogeoquímicas (Míguez, et al., 2001), fueron luego adoptadas en el marco de la Comisión Asesora en Agua y Saneamiento (COASAS) como unidad de gestión de cuencas, junto con las de agua superficial principales, ya que por más que son grandes reservas de agua, los acuíferos no están exentos de problemas de sobre-explotación ni de riesgos de contaminación. Así por ejemplo, el Sistema Acuífero Guaraní, reservorio subterráneo de agua transfronterizo que Uruguay comparte con Argentina, Brasil y Paraguay, de importancia estratégica mundial, según Gómez et al. (2010) está siendo intensamente explotado para provisión de agua potable, usos industriales y de la agricultura, por lo cual se deben estudiar las tasas de recarga. El Sistema Acuífero Guaraní fue uno de los primeros en tener representantes en su organismo de gestión en la estructura nacional de las cuencas consideradas.

Mediante la modificación del artículo número 47 de la Constitución de la República, plebiscitada en octubre de 2004, se consagra el derecho de todos los habitantes del país al agua y al saneamiento (Uruguay, 2004). En el proceso actual se da participación a todos los actores, incluyendo a los gobiernos locales, municipales, lo cual es aun más importante dado el proceso de descentralización. Actualmente la GIRH está abarcando incluso niveles individuales, de vecinos interesados u ONGs, dependiendo del tamaño de la cuenca.

La Ley Reglamentaria del inciso $2^{\circ}$ del artículo 47 de la Constitución de la República (Uruguay, 2004), creó la Dirección Nacional de Agua y Saneamiento, DINASA, actualmente DINAGUA, en la órbita del MVOTMA, la cual, trabajando en conjunto con la COASAS, constituida por referentes con competencia en la gestión y control de la calidad del agua tanto a nivel nacional como municipal, elevó a consideración del Parlamento Nacional el texto que resume los principios rectores de la política de aguas en nuestro país, Ley 18.610 «Política Nacional de Aguas» (Uruguay, 2009).

Formalmente, la COASAS comenzó a trabajar en mayo de 2007 en la elaboración del anteproyecto de esta ley que consta de 12 capítulos y que hace hincapié en principios de equidad, asequibilidad, solidaridad y sustentabilidad, remarcando que la gestión debe ser integral, transversal y participativa, y al mismo tiempo destina recursos a la capacitación y la investigación científica y tecnológica en materia de aguas. Se crea el Consejo Nacional de Agua, Ambiente y Territorio, integrado por representantes del gobierno, usuarios y sociedad civil, cada uno de ellos con igual representación.

Se formaron los Consejos Regionales de Recursos Hídricos, integrados por representantes del gobierno, usuarios y sociedad civil. En su artículo 25 la ley enuncia que «a los Consejos Regionales de Recursos Hídricos compete planificar y deliberar sobre todos los temas relativos al agua en la región, tal como la formulación de Planes Regionales de Recursos Hídricos». Establece también que los usuarios que comprometan el ciclo hidrológico se encarguen de realizar 
monitoreos sistemáticos de las fuentes de agua de manera que puedan generarse bases de datos públicas.

Para la gestión se priorizaron tres regiones: cuenca del Río Uruguay, Laguna Merín y Río de la Plata y su frente marítimo. En el artículo 28, la ley establece que los Consejos de estas regiones «promoverán y coordinarán la formación de Comisiones de Cuencas y Acuíferos que permitan dar sustentabilidad a la gestión local de los recursos naturales y administrar los potenciales conflictos por su uso». Su rol es asesor de los Consejos Regionales, y su integración, representativa de la presencia de actores locales activos en el territorio.

En base a lo dispuesto por la Ley 18.610 (Uruguay, 2009), se delinea un plan como herramienta para desarrollar la Política de Aguas que marca el artículo 1 de la ley (Ministerio de Vivienda Ordenamiento Territorial y Medio Ambiente, 2011).

Actualmente están en funcionamiento los Consejos Regionales de Recursos Hídricos para la cuenca del Río Uruguay con comisiones de cuenca para los ríos Cuareim, Tacuarembó, para el Sistema Acuífero Guaraní y para el Arroyo San Antonio. Se encuentra en proceso la conformación de la comisión de cuenca del Río Cebollatí, en el ámbito del Consejo Regional de la Laguna Merín. El Consejo Regional para la cuenca del Río de la Plata y su frente marítimo tiene en funcionamiento las comisiones de cuenca del Río Santa Lucía, de la Laguna del Sauce y de la del Cisne (Saravia, et al., 2014).

\section{Herramientas y tecnologías requeridas para la sostenibilidad de los procesos productivos}

La agricultura es responsable del 70\% del uso de agua a nivel mundial. De modo que un planeamiento integrado que contemple todos los posibles usos de la tierra resulta fundamental, así como también el de los recursos hídricos y la mejora en el desempeño de los servicios de provisión de agua potable, todo lo cual requiere conocer la cuenca para estar alerta sobre los peligros de sequía, de inundación, de contaminación química, física o microbiológica.

\subsection{Mapas de peligros y de riesgos}

En opinión del autor, para conocer las cuencas es muy útil generar mapas de riesgos de exceder límites de nutrientes o mostrar los riesgos de contaminación o eutrofización a nivel macro, continental, a cuencas y sub-cuencas. Estos instrumentos son herramientas modernas para la GIRH y representan las áreas donde hay mayor probabilidad de inundaciones, sequías o la condición que impida cumplir con una normativa en parámetros tales como nutrientes o contaminantes.

Los mapas de riesgos identifican y jerarquizan riesgos de eventos extremos debidos al cambio climático, tales como inundaciones o sequías (Tkhilava, 2015) o contaminación, representados en forma geográficamente referenciada. Estos mapas son utilizados por instituciones que generan indicadores globales (Gassert, 2013) y también aplicados exitosamente en Europa por las agencias ambientales (European Environmental Agency, 2010) y en otras cuencas a nivel mundial.

\subsection{Sistemas de apoyo a las decisiones}

Los monitoreos, basados en sistemas de apoyo a las decisiones o decision support systems (DSS) (Flügel, 2010), son una herramienta recomendable para gestión de cuencas. Sirven para el nivel de gestión de la organización y ayudan a tomar decisiones en escenarios que pueden estar cambiando rápidamente y cuyos resultados no pueden ser anticipados con facilidad (IWA WaterWiki). Se sustentan sobre la base de programas informáticos tales como

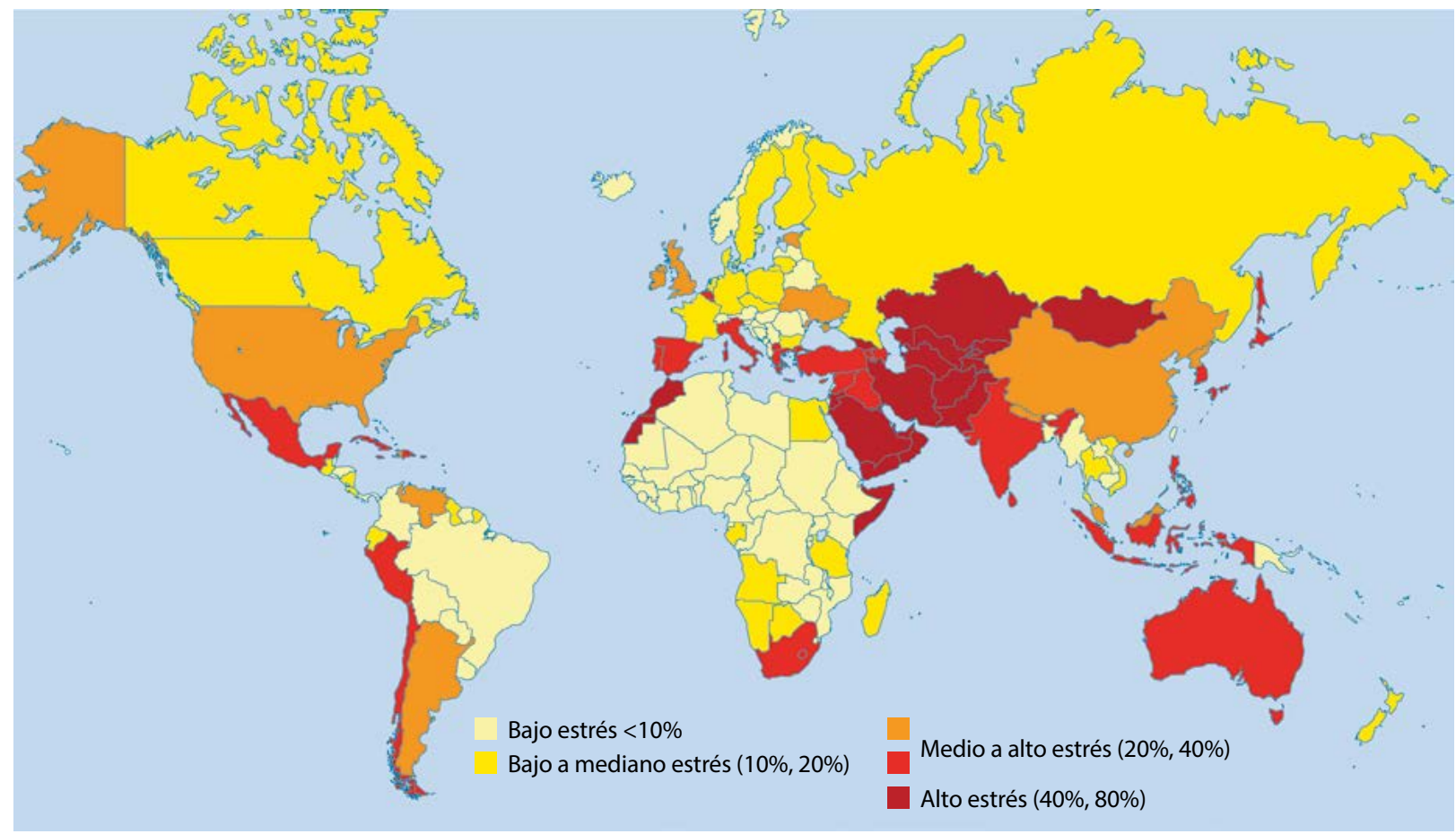

Figura 1. Indicador de estrés hídrico en el mundo. Modificado de Aqueduct, World Resources Institute, WRI, 2013. 
BASINS (Better Assessment Science Integrating point \& Non-point Sources), utilizado en Estados Unidos, en tanto en Europa también se desarrollan sistemas similares para implementar la Directiva Marco del Agua.

Las DSS basadas en el conocimiento permiten gestionar el suministro del recurso agua y mejorar la eficiencia de la energía hacia sistemas de bombeo usados para distribución del agua, para satisfacer ambas demandas esperadas. Comprenden el ciclo del agua desde una perspectiva de gestión.

Estos sistemas se basan en tecnologías de la información (TIC) y mapas de riesgos para presentar a los generadores de políticas y a las empresas potabilizadoras las consecuencias de inundaciones, por ejemplo, pero son expandibles con análisis de contaminación de agua y permiten de esa manera localizar la ubicación de las tomas de agua más vulnerables al ingreso de sustancias peligrosas o bacterias. Además de los mapas de riesgos se incluyen también los de energía, recursos energéticos, combustibles, telecomunicaciones, redes, aspectos financieros, seguridad alimentaria, cuidado de la salud, transportes, etcétera, que pueden ser afectados durante una inundación (Kwiecień y Walczykiewicz, 2015).

\subsection{Monitoreos ambientales}

En este contexto, los monitoreos sirven para diagnosticar el estado de situación de los recursos, dar continuidad al control y a la investigación científica, evaluar la marcha de las acciones emprendidas, y decidir las nuevas actividades a fin de disminuir los riesgos de afectar la biodiversidad y la disponibilidad del agua para fines económicos y sanitarios. Ejemplos de estas aplicaciones se encuentran en los programas de monitoreo de la biodiversidad en el Río Rín (Senckenberg, 2015).

\subsection{Eco-innovación}

Las políticas ambientales para tender a la sostenibilidad se apoyan no solo en tratados internacionales y en la cooperación, sino también en empresas eco-innovadoras (Ekins, 2010). Algunas de las tecnologías innovadoras implican el reúso del agua mediante métodos de depuración especiales (tecnologías de oxidación avanzada, reactores biológicos de membrana de ultrafiltración para remoción de contaminantes químicos y microbiológicos convencionales y emergentes) y la conservación y el ahorro de agua, como en el caso de la cosecha de agua de lluvia.

\subsection{Tecnologías inteligentes}

El vínculo entre las tecnologías de la información y las comunicaciones (TIC) y la gestión del agua se evidencia en Smart Water, un término que abarca metodologías robustas, prácticas y accesibles económicamente para gestionar el agua y que se apoya en el acceso a grandes bases de datos o big data. Las formas de implementarlo pueden incluir desde medidores de agua inteligentes hasta aplicaciones virtuales y sistemas informáticos de apoyo a las decisiones (Savić, et al., 2014).

\subsection{Nuevas tendencias en indicadores}

A nivel macro se podría aplicar el concepto de la huella de agua, un indicador del uso del agua en relación al consumo definido como el volumen de agua necesario para la producción de bienes y servicios consumidos por los habitantes de un país (Hoekstra y Chapagain, 2007).

El comercio del agua virtual permite diferenciar entre niveles locales, regionales y globales y vincularlos a la GIRH. Para efectuar una gestión ambiental sostenible del agua en América Latina y el Caribe se deben cuantificar los recursos y evaluarlos en forma exhaustiva (Mekonnen, et al., 2015). También es necesario realizar la evaluación de la porción recuperable en forma de reúso de agua y la dependencia de sus múltiples usuarios para generar políticas de gestión de recursos hídricos. Estas herramientas han demostrado ser útiles para informar las decisiones estratégicas que puedan afectar el uso del agua porque conllevan información sobre sostenibilidad hidrológica
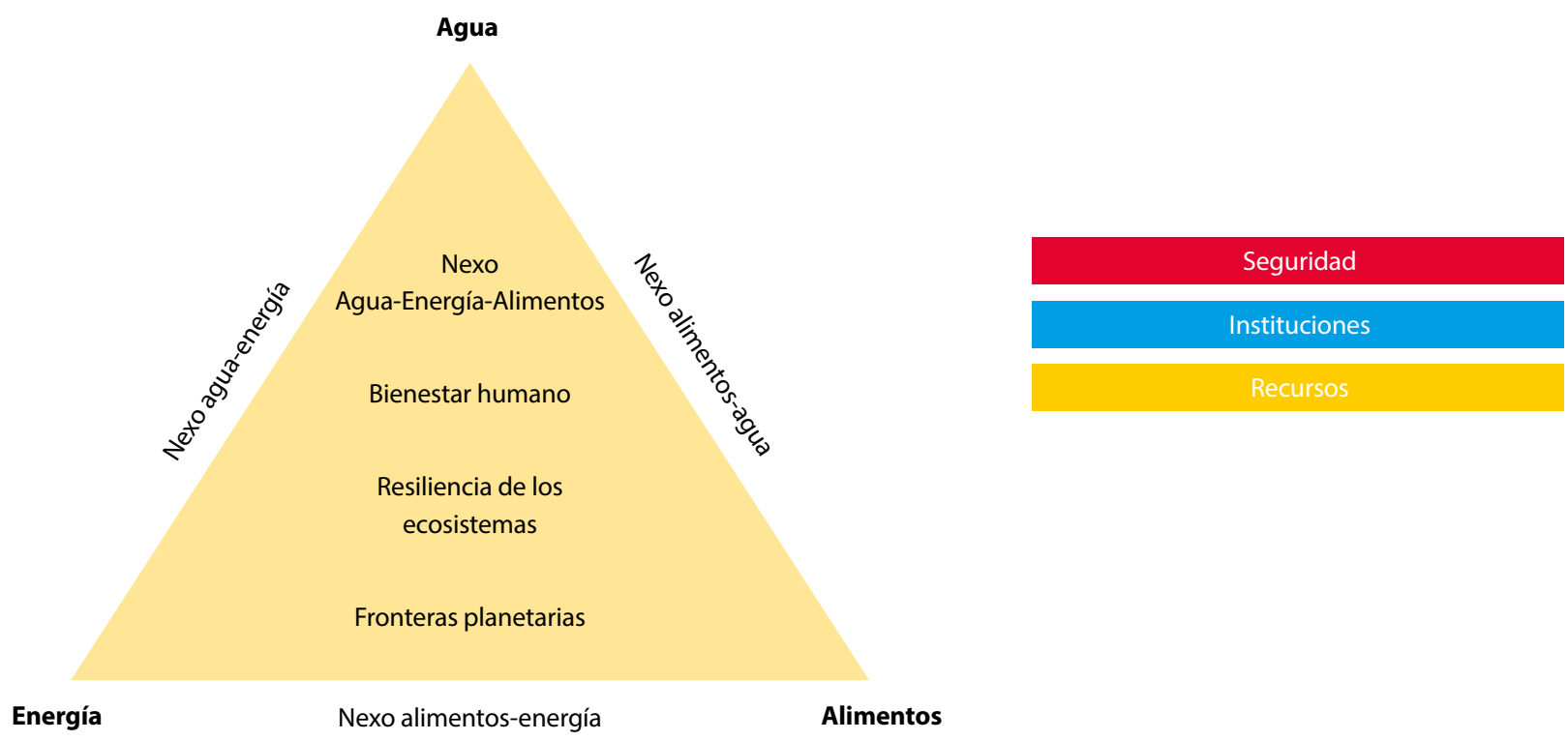

Figura 2. Enfoque del nexo entre agua, energía y alimentos, y sus interrelaciones. Modificado de Scott, et al., 2015. 
y prioridades políticas en cuanto a comercio, desarrollo económico y bienestar social (Chapagain y Tickner, 2012).

Los mapas de riesgos globales, según Gassert et al. (2013a), muestran la distribución del indicador de estrés hídrico a nivel mundial (Figura 1).

Hay otros indicadores, tales como los de reconocimiento de sequía (RDI por su sigla en inglés), que pueden ser utilizados para evaluar cambio climático y acoplar este concepto a la GIRH (Shokoohi y Morovati, 2015). También se ha desarrollado un conjunto de indicadores ecológicos que permiten evaluar la integridad del ecosistema (Parr, et al., 2015).

De acuerdo a Brand (2012), la «economía verde» falla al no tener en cuenta las restricciones políticas, económicas y culturales pero es atractiva para actores socioeconómicos y para capitales financieros que ven la protección ambiental como un campo de inversión nuevo.

Recientemente, se alude a los enfoques adaptativos que vinculan el nexo agua-energía-alimentos, concepto adoptado por complejas cadenas de suministro mundial para gestión de riesgos, para evaluar estratégicamente las decisiones que puedan afectar los compromisos competitivos en forma interrelacionada entre agua, energía y alimentos y, especialmente, en la situación actual de cambio climático (Scott, et al., 2015) (Figura 2).

De acuerdo a Petersen-Perlman et al. (2012), la seguridad del agua es la disponibilidad del agua dulce en la correcta cantidad y calidad, en todo momento, para sistemas dependientes, como prerrequisito para la seguridad humana y ambiental y el crecimiento económico. En especial, es un desafío en el caso de los recursos de agua transfronterizos. En su análisis sobre gestión de cuencas e institucionalidad, estos autores argumentan que los sistemas con alta capacidad institucional y baja tasa de cambio tienen más probabilidad de poseer resiliencia hidropolítica.

En esta línea de pensamiento, dado que las áreas urbanas son consumidoras de materiales y energía, se pueden aplicar evaluaciones estratégicas con técnicas tales como el Análisis de Sistemas Multisectorial para estimar los flujos de recursos entrantes, circulantes y salientes (nutrientes, agua y energía), incluyendo el estudio de sinergias, antagonismos y beneficios económicos de las tecnologías innovadoras para su correcta selección.

\section{Discusión}

Algunos autores tales como Leendertse et al. (2008) opinan que desde el punto de vista ecosistémico si el proceso es «top-down», con poco involucramiento de grupos de interés, los cambios no se verán, teniendo en cuenta, además, que el medio ambiente es el que menos se considera cuando se tratan las políticas de gestión del agua.

En países en desarrollo existe una falta de armonización de tecnologías de la información y sistemas georreferenciados, pero esto puede ser solucionado; Tailandia, por ejemplo, se basó en tecnología «open-source» para publicar los datos hidrometeorológicos en internet.

Como se menciona en la sección 4, otras tecnologías a implementar son los sistemas de apoyo a las decisiones. La utilidad de las DSS para su uso en la GIRH se evidencia en las ventajas que otorga a la hora de tomar decisiones estratégicas con respecto a las mejores opciones técnicas. Aydin et al. (2015) presentan un caso de aplicación en el que un sistema de distribución de agua fue evaluado por medio de técnicas computacionales en base a índices de sostenibilidad, utilizando la confiabilidad, la resiliencia y la vulnerabilidad como criterios de desempeño que se hicieron visibles en forma de diagramas para facilitar la lectura por los tomadores de decisiones.

El concepto de huella hídrica o huella del agua, «water footprint» en inglés, fue introducido por Hoekstra (2003) y posteriormente desarrollado por Hoekstra y Chapagain (2007) para proveer un marco para analizar el vínculo entre el consumo humano y la apropiación del agua dulce global. La huella de agua azul refiere al volumen de agua superficial y subterránea consumida (evaporada) como resultado de la producción de un producto (Mekonnen y Hoekstra, 2011). Según Gassert et al. (2013a, 2013b), en cuanto a disponibilidad de aguas azules Uruguay aparece en un lugar privilegiado en el contexto internacional, pero el indicador de riesgos de inundaciones es alto. El indicador de estrés hídrico basal preliminar desarrollado por estos autores ubica a las cuencas de Uruguay en el puesto 121. Este puesto se obtiene en base al cálculo que considera el total anual de uso del agua como un porcentaje del disponible anual de aguas azules; en la escala los valores más altos indican la competencia mayor entre usuarios, con un puntaje de 0.86 (desviación estándar 0.91) para Uruguay, equivalente a bajo estrés, tendiente a mediano.

Algunos conceptos como el de la huella de agua tampoco están tan lejos de nuestra realidad; de hecho, fue aplicado por Mekonnen et al. (2015) para la región de América Latina y el Caribe, incluyendo a Uruguay, por lo cual a futuro se puede constituir en un factor determinante a la hora de exportar nuestras commodities, es decir, nuestros productos básicos y sin gran valor agregado.

Los indicadores de sostenibilidad están siendo desarrollados por equipos de investigadores nacionales para evaluar el impacto de actividades productivas tales como las agropecuarias por pastoreo o en forma confinada (Modernel, et al., 2013). Es posible continuar refinando los estudios introduciendo conceptos ecológicos tales como la distribución de la sensibilidad de las especies acuáticas o terrestres a determinado nivel de contaminación (Species sensitivity distributions, SSDs), que a su vez se pueden incorporar en evaluaciones de riesgos (Míguez, 2013) y así comparar los riesgos de introducir un tratamiento por pesticidas, por ejemplo.

\section{Conclusiones y recomendaciones}

A nivel nacional las estructuras legal e institucional ya existen y actualmente están en proceso de implementación las prácticas que permitan ser más eficientes en la ejecución de proyectos. La asignación de recursos nacionales debería encaminarse a fortalecer este proceso, así como a generar herramientas de integración de la información existente, tales como bases de datos robustas que sean de fácil acceso a los investigadores, tomadores de decisión y el público en general. El sistema nacional todavía presenta algunos problemas de comunicación entre las instituciones, pero hay un avance sustancial en este sentido con respecto a años anteriores.

Gestionar bien los recursos hídricos beneficia tanto al ser humano como a la naturaleza, de modo que la Gestión Integrada de Recursos Hídricos debería proveer soluciones. La razón por la cual es necesario generar proyectos demostrativos a nivel de 
una cuenca, como los proyectos de demostración en cuencas, es que las acciones concretas e innovadoras de gestión, herramientas y tecnología construyen confianza para que los tomadores de decisiones basen sus políticas nacionales y su planeamiento en la seguridad de que el sistema funcionará. Acoplar los planes de monitoreo a la estructura de las comisiones de cuencas y comités regionales permite nutrir a las partes interesadas con la información de quienes la manejan más directamente, y destinar recursos del país a la generación de proyectos alineados con las necesidades que surjan de estos foros.

La implementación de las políticas y la gestión se debería basar en cifras, datos y hechos. Lo cual alude, en definitiva, a concretar el espíritu de la Ley 18.610 (Uruguay, 2009), en cuanto al acceso público a bases de datos actualizados continuamente como gran desafío a lograr en los próximos años. Asimismo, supone informar las decisiones por medio de evaluaciones de riesgos y tecnologías de modelación, aplicaciones de las TIC a la gestión del agua, e indicadores de sostenibilidad y de la huella de agua, para bien del ambiente, el comercio internacional, la salud de la comunidad y el progreso de nuestro país.

\section{Referencias}

Anzaldi, G. Rubion, E., Corchero, A., Sanfeliu, R., Domingo, X., Pijuan, J., Tersa, F., 2014. Towards an Enhanced Knowledge-based Decision Support System (DSS) for Integrated Water Resource Management (IWRM). 16th Water Distribution System Analysis Conference, WDSA2014 Urban Water Hydroinformatics and Strategic Planning. En: Procedia Engineering, 89, pp.1097-1104.

Aven, T. y Renn, O., 2009. On risk defined as an event where the outcome is uncertain. En: Journal of Risk Research, 12(1), pp.1-11.

Aydin, N.Y., Zeckzer, D., Hagen, H. y Schmitt, T., 2015. A decision support system for the technical sustainability assessment of water distribution systems. En: Environmental Modelling \& Software, 67, pp.31-42.

Brand, U., 2012. Green economy - The next oxymoron? No lessons learned from failures of implementing sustainable development. En: GAIA Ecological Perspectives for Science and Society, 21(1), pp.28-32.

Chapagain, A.K. y Tickner, D., 2012. Water footprint: help or hindrance? En: Water Alternatives, 5(3), pp.563-581.

Díaz Ortiz, J.E. y Freire Delgado, B., 2008. Manejo del recurso hídrico en culturas precolombinas [En línea]. En: EIDENAR, 7. [Consulta: 15 de noviembre de 2015]. Disponible en: http://revistaeidenar.univalle.edu.co/ revista/ejemplares/7/j.htm.

DINAGUA, 2012. Regionalización y correlaciones de parámetros hidrológicos [En línea]. Montevideo: MVOTMA. [Consulta: 7 de setiembre de 2012]. Disponible en: http://www.mvotma.gub.uy/ ciudadania/item/10003084-regionalizaci\%C3\%B3ny-correlaci\%C3\%B3n-de-par\%C3\%Almetroshidrol\%C3\%B3gicos.html

Dirección Nacional de Hidrografía, 2015. Disponible en: http://www.puertosdeportivos.com.uy/dnh/index.php

Duek, A.E. y Comellas, E.A., 2011. Ordenamiento territorial y gestión integrada de los recursos hídricos: dos políticas implementadas en Argentina. En: Tiempo y Espacio, 26, pp.153-170.
Ekins, P., 2010. Eco-innovation for environmental sustainability: concepts, progress and policies. En: International Economy and Economical Policy, 7, pp.267-290.

European Environmental Agency, 2010. Exceedance of critical loads for eutrophication due to the deposition of nutrient nitrogen in 2010 [En línea]. [s.l.]: EEA. [Consulta: 21 de octubre de 2015]. Disponible en: http://www.eea.europa. eu/data-and-maps/figures/exceedance-of-critical-loadsfor-eutrophication-due-to-the-deposition-of-nutrientnitrogen-in-2010

Flügel, W-A., 2009. Applied geoinformatics for sustainable IWRM and climate change impact analysis. En: Technology, Resource Management \& Development, 6, pp. 57-85.

Gassert, F., Landis, M., Luck, M., Reig, P. y Shiao, T., 2013a. Aqueduct global maps 2.0. Working Paper [En línea]. Washington: World Resources Institute. [Consulta: 24 de febrero de 2015]. Disponible en: http://www.wri.org./ publication/aqueduct-metadata-global.

Gassert, F., Reig, P., Luo, T. y Maddocks, A., 2013b. Aqueduct country and river basin rankings: a weighted aggregation of spatially distinct hydrological indicators. Working paper [En línea]. Washington: World Resources Institute. [Consulta: 24 de febrero de 2015]. Disponible en: http://wri.org/ publication/aqueduct-country-river-basin-rankings.

Global Water Partnership, 2012. The enabling environment (A) [En línea]. Stockholm: GWP [Consulta: 14 de febrero de 2015]. Disponible en: http://www.gwp.org/en/ToolBox/ TOOLS/The-Enabling-Environment/

Global Water Partnership Technical Advisory Committee (TAC), 2000. Integrated water resource management. Stockholm: GWP. (TAC Background Papers No. 4).

Gómez, A.A., Rodríguez, L.B. y Vives, L.S., 2010. The Guarani Aquifer system: estimation of recharge along the UruguayBrazil border. En: Hydrogeology Journal, 18, pp.1667-1684.

Grigg, N.S., 2008. Integrated water resources management: balancing views and improving practice. En: Water International, 33(3), pp.279-292.

Hoekstra, A.Y. (ed.), 2003. Virtual water trade: Proceedings of the International Expert Meeting on Virtual Water Trade, Delft, The Netherlands, 12-13 December 2002, Value of Water Research Report Series No.12, IHE, Delft, the Netherlands.

Hoekstra, A.Y. y Chapagain, A.K., 2007. Water footprints of nations: water use by people as a function of their consumption pattern. En: Journal of Water Resources Management, 21, pp.35-48.

Iza, A. y Stein, R. (eds), 2009. RULE - Reforming water governance. Gland: IUCN.

Kwiecień, M. y Walczykiewicz, T., 2015. Decision Support System for flood protection of critical infrastructure facilities based on water intake map. En: Polish Journal of Environmental Studies, 24(4), pp.1649-1657.

Leendertse, Kees, Mitchell, Steve y Harlin, Joakim, 2008. IWRM and the environment: a view on their interaction and examples where IWRM led to better environmental management in developing countries. En:Water SA, 34(6), pp.691-698.

Lofrano, G., Brown, J. y De Feo, G., 2012. Water pathways through the ages: from early aqueducts to next generation of wastewater treatment plants. En: Sharma, S.K. y Sanghi, R., (eds). Advances in water treatment and pollution prevention. Rotterdam: Springer. pp.37-54.

Mekonnen, M. M. y Hoekstra, A.Y., 2011. The green, blue and 
grey water footprint of crops and derived crop products. En: Hydrology and Earth Systems Science, 15, pp.1577-1600. Mekonnen, M.M., Pahlow, M., Aldaya, M.M., Zarate, E. y Hoekstra, A.Y., 2015. Sustainability, efficiency and equitability of water consumption and pollution in Latin America and the Caribbean. En: Sustainability, 7, pp.2086-2112.

Míguez, D., di Candia, C., Baklayan, P., Agüero, S., Hourdebaigt, R., Massa, E. y Carrión R., 2001. Composición típica de las aguas subterráneas del Uruguay por provincias hidrogeológicas. En: AOAC International. IV Simposio y Exposición de la Sección de América Latina y el Caribe de AOAC International: desafíos analíticos para el nuevo milenio. Montevideo: AOAC Internacional pp.1-10.

Míguez, D., 2013. Integrated risk assessment of endocrine disruptors in the Uruguay River [En línea]. Cranfield: Cranfield Water Science Institute, School of Applied Sciences, Cranfield University, Reino Unido. (Tesis de doctorado). Disponible en: https://dspace.lib.cranfield. ac.uk/handle/1826/8201

Ministerio de Vivienda, Ordenamiento Territorial y Medio Ambiente, Dirección Nacional de Aguas, 2011. Hacia un plan de gestión integrada de recursos hídricos. Agenda para la acción [En línea]. [Consulta: 2 de marzo de 2015]. Disponible en: http://www.mvotma.gub.uy/component/ k2/item/10002981.html?Itemid=100185

Modernel, P., Astigarraga, L. y Picasso, V., 2013. Global versus local environmental impacts of grazing and confined beef production systems. En: Environmental Research Letters, 8(3), 035052.

Naciones Unidas, 2003. Gestión integrada de recursos hídricos (GIRH). Decenio internacional para la acción [En línea]. [s.l.]: UN. [Consulta: 14 de febrero de 2014]. Disponible en: http://www.un.org/spanish/waterforlifedecade/iwrm.shtml.

Naciones Unidas, 2012. The UN-Water status report on the application of integrated approaches to water resources management [En línea]. [s.1.]: UN. [Consulta: $17 \mathrm{de}$ febrero de 2014]. Disponible en: http://www.un.org/ waterforlifedecade/pdf/un_water_status_report_2012.pdf

Naciones Unidas, 2014a. A post-2015 global goal for water: synthesis of key findings and recommendations from $U N$ Water [En línea]. [s.l.]: UN. [Consulta: 17 de febrero de 2014]. Disponible en: http://www.unwater.org/fileadmin/ user_upload/unwater_new/docs/Topics/UN-Water_ paper_on_a_Post-2015_Global_Goal_for_Water.pdf

Naciones Unidas, 2014b. Agua y energía. Informe de las Naciones Unidas sobre el desarrollo de los Recursos Hídricos en el Mundo 2014 [En línea]. [s.l.]: UN. [Consulta: 26 de enero de 2014]. Disponible en: http://unesdoc.unesco.org/ images/0022/002269/226961S.pdf

Naciones Unidas, 2015. UN-water's recommendations for a global goal on water [En línea]. [s.l.]: UN. [Consulta: 20 de febrero de 2015]. Disponible en: http://www.unwater.org/ topics/water-in-the-post-2015-development-agenda/newsand-updates/en/

Novel Integrated Water NOVIWAM. Proyecto (http://www. redotriandalucia.es/catalog/novel-integrated-wat-68/)

Organisation for Economic Co-Operation and Development (OECD), Comisión Económica de las Naciones Unidas para Europa. Integrated Water Resources Management in Eastern Europe, the Caucasus and Central Asia, European Union Water Initiative National Policy, 2014. Dialogues progress report 2013 [En línea]. New York: UN. [Consulta 20 de febrero de 2015]. Disponible en: http://www.unece.
org/fileadmin/DAM/env/water/publications/NPD_ IWRM_study/ECE_MP.WAT_44_en.pdf.

Parr, T. B., Cronan, C.S., Danielson, T.J., Tsomides, L. y Simon, K.S., 2016. Aligning indicators of community composition and biogeochemical function in stream monitoring and ecological assessments. En: Ecological Indicators, 60, pp.970-979.

Petersen-Perlman, J.D., Veilleux, J.C., Zentneer, M. y Wolf, A.T., 2012. Case studies on water security: analysis of system complexity and the role of institutions. En: Journal of Contemporary Water Research \& Education, 149, pp.4-12.

Porto, M.F.A. y La Laina Porto, R., 2008. Gestão de bacias hidrográficas. En: Estudos avançados, 22(63), pp.43-59.

Presidencia de la República Oriental del Uruguay, 2013. PLANAGUA. Gobierno apoya gestión participativa de recurso hídrico y conciencia colectiva de su uso [En línea]. Montevideo: Presidencia de la República. [Consulta: 17 de febrero de 2014]. Disponible en: http://presidencia. gub.uy/comunicacion/comunicacionnoticias/planaguagobernanza-dinagua-mvotma-gonzalez

Programa de las Naciones Unidas para el Medio Ambiente, 2014. Towards integrated water resources management: international experience in development of river basin organization [En línea]. Amarat: UNETP. [Consulta: 13 de noviembre de 2015]. Disponible en: http://www.unep. org/disastersandconflicts/portals/155/countries/Sudan/ pdf/SudanWRM.pdf

Programa Mundial de Evaluación de los Recursos Hídricos. Secretaria del Programa Mundial de Evaluación de los Recursos Hídricos. División de Ciencias del Agua, UNESCO, Naciones Unidas, 2014. Agua y energía. Informe de las Naciones Unidas sobre el desarrollo de los recursos hídricos en el mundo 2014 [En línea]. Perugia: UNESCO. [Consulta: 21 de enero de 2015]. Disponible en: http://unesdoc.unesco.org/ images/0022/002269/226961S.pdf

Real Academia Española, 2001. Diccionario de la lengua española [En línea]. 22a ed., con agregados hasta 2012. Madrid: RAE. [Consulta: 24 de junio de 2015]. Disponible: http://lema.rae.es/drae/?val=gobernza.

Rossi, G., 2015. Achieving ethical responsibilities in water management: A challenge. En: Agricultural Water Management, 147, pp.96-102.

Santos Pereira, L., Cordery, I. y Iacovides, I., 2009. Coping with water scarcity. Addressing the challenges. Rotterdam: Springer.

Saravia, M, Panizza, A. y González, D., 2014. Consejos regionales de recursos hídricos y comisiones de cuencas y acuíferos. (Presentación en ppt de la sesión de COASAS para difusión del Informe Anual de actividades de la Dirección Nacional de Aguas, Período 2013-2014).

Savić, D, Vamvakeridou-Lyroudia, L. y Kapelan, Z, 2014. Smart meters, smart water, smart societies: The iWIDGET Project. En: Procedia Engineering, 89, pp.1105-1112.

Scott, C.A., Kurian, M. y Wescoat Jr, J.L., 2015. The water-energy-food nexus: enhancing adaptive capacity to complex global challenges. Chapter 2. En: Kurian, M. y Ardakanian, R., (eds.). Governing the Nexus. Rotterdam: Springer.

Senckenberg Research Institute and Natural History Museum Frankfurt, 2015. [Consulta: 21/10/2015]. Disponible en: http://www.senckenberg.de/root/index.php?page_ $\mathrm{id}=71 \&$ PHPSESSID=digq5mfchvnvjfrcha0ge7pik2. Shokoohi, A. y Morovati, R., 2015. Basinwide comparison 
of RDI and SPI within an IWRM framework. En: Water Resources Management, 29(6), pp.2011-2026.

Szreter, S., 1997. Economic growth, disruption, deprivation, disease, and death: On the importance of the politics of public health for development. En: Population and Development Review, 23(4), pp.693-728.

Tkhilava, N., 2015. Importance of integrated water resources management in flood and flash flood management. En: American Journal of Environmental Protection, 4(3-1), pp.8-13.

Uruguay. Poder Legislativo, 2004. Constitución de la República. Constitución 1967 con las modificaciones plebiscitadas el 26 de noviembre de 1989, el 26 de noviembre de 1994, el 8 de diciembre de 1996 y el 31 de octubre de 2004 [En línea]. Montevideo: Poder Legislativo. [Consulta: 27 de febrero de 2015]. Disponible: http://www.parlamento.gub.uy/ constituciones/const004.htm.

Uruguay. Ley 18.610, de 02 de octubre de 2009. Diario Oficial,
28 de octubre de 2009, No. 27845, p.1038.

Uruguay. Decreto 349/005, de 21 de setiembre de 2005. Diario Oficial, 3 de octubre de 2005, p.700.

Water Economic Forum, 2015. The global risks report 2015 [En línea]. 10a ed. Geneva: WEF. [Consulta: 26 de febrero de 2015]. Disponible en: http://www.weforum.org/reports/ global-risks-report-2015

While, M., 2015. The Industrial Revolution. Georgian Britain. The British Library. [Consulta: 23/2/2015]. Disponible en: http://www.bl.uk/learning/histcitizen/georgians/ industrial/industrialisation.html.

World Health Organization, 2004. International Programme on Chemical Safety, IPCS risk assessment terminology [En línea]. Geneva: WHO. (IPCS harmonization project; document $\mathrm{N}^{\circ}$ 1). pp.13. [Consulta: 6 de octubre de 2015]. Disponible en: http://www.who.int/ipcs/methods/ harmonization/areas/ipcsterminologyparts1and2. pdf?ua $=1$. 